\title{
Design considerations to realize differential absorption based optofluidic sensors for determination of ionic content in water
}

\author{
Gerrit W. Steen, ${ }^{\mathrm{a}, \mathrm{b}, \dagger}$ Elmar C. Fuchs $^{\mathrm{b}}$, Adam D. Wexler ${ }^{\mathrm{b}}$, and Herman L. Offerhaus ${ }^{\mathrm{a}}$
}

\begin{abstract}
Integrated optofluidic absorption spectroscopy has the potential to be used in the real-time identification and quantification of ionic content in drinking water. Such an approach requires single mode operation in combination with low propagation and bend losses. The design criteria for the realization of an integrated Near-infrared (NIR) sensor platform fabricated using silicon waveguide based technology is presented. The cross-section of TriPleX waveguides was optimized for a spectral region between 940 and $1040 \mathrm{~nm}$. The waveguide structure provides single mode operation, high sensitivity combined with reasonable tolerance for fabrication deviations and $<0.01 \mathrm{~dB} / \mathrm{cm}$ bend losses for a radius of $500 \mu \mathrm{m}$. The optimum sensing length on chip was determined for propagation losses of $0.1 \mathrm{~dB} / \mathrm{cm}, 0.5 \mathrm{~dB} / \mathrm{cm}$ and $1.5 \mathrm{~dB} / \mathrm{cm}$. Furthermore, a $\Xi$ coupler was designed for the spectral region of interest $(0.94$ to $1.04 \mu \mathrm{m})$. Multiple integrated photonic circuit layouts varying in functionality and complexity are reviewed. These optofluidic absorption sensors offer in-situ \& in-line capabilities, potentially low-cost, remote signal data processing, and mechanical \& thermal stability. This work provides a roadmap for maximizing the sensitivity of broadband spectroscopy using differential absorbance.
\end{abstract}

Index Terms - Absorption, Integrated design, Integrated optics, Optical interferometry, Optimization methods, Optical sensors, Sensitivity, Silicon photonics

\section{INTRODUCTION}

$\mathrm{R}$ EGULATORY AGENCIES such as the world health organization (WHO) and the environmental protection agency (EPA) are continuously tightening the acceptable drinking standard as well as their reporting requirements. Inorganic ionic are relevant for general safety for water consumption. Some ions (e.g., $\mathrm{Pb}^{2+}, \mathrm{Hg}^{2+}, \mathrm{Cd}^{2+}, \mathrm{Ti}^{3+}, \mathrm{As}^{3+}$ ) can even be toxic in trace amounts [1]. The concentration of these ions should be monitored in real-time to avoid natural poisoning, as well as bioterrorism [2]. The use of an optofluidic sensor can meet these demands.

The standard technology to determine the ionic content in water is ion chromatography (IC) [3]. Time intensive and required dedicated expertise are known disadvantages of such an approach. Though IC on-chip devices are currently being

a. University of Twente, Optical Sciences, P.O. Box 217, 7500AE Enschede, The Netherlands.

b. Wetsus - European centre of excellence for sustainable water technology, Oostergoweg 9, 8911MA Leeuwarden, The Netherlands.

$\dagger$ Corresponding author: gerwin.steen@wetsus.nl developed [4]. Integrated optofluidic devices have a number of advantages over free space systems and are well suited for insitu/in-line, real-time monitoring of water quality [5].

In such a sensor both the fluidic and optical parts are miniaturized (micro scale) and combined, ensuring on-/in line capabilities. The optical part of the sensor meets the real-time requirement. The microfluidic part is essential to probe the fluid. Part of the energy in the evanescent field of the optical mode is absorbed. Planar waveguides are positioned on the bottom of the microfluidic channels. Interaction with the fluid is enabled in the sensing window by removal of the protecting upper cladding layer (Fig. 1). The TriPleX platform allows a high evanescent field sensitivity in combination with low bend losses.

Low-loss light propagation through optofluidic devices is limited to the visible and near-infrared (VIS/NIR, 0.4 to 2.6 $\mu \mathrm{m})$ wavelength region. Direct detection of most mono-atomic ionic content in water is not feasible with a light source having these wavelengths. The interaction of light with ions of most dissolved inorganic salts is orders of magnitude lower than the interaction of its complex water environment (exceptions are transition metal ions). The aqueous absorption spectrum can be probed in the VIS/NIR wavelength regions and is known to be uniquely influenced by the presence of anions and cations [6]-[9].

Previous work showed a potential utilization of a spectral window ranging from 0.94 to $1.04 \mu \mathrm{m}$ for the identification and quantification of electrolytes based on their specific perturbations of the water absorption spectrum [10],[11]. The same optical method can be applied to integrated optofluidic chips. Sensitivity to fluid in the microfluidic channels is achieved by removal of the top cladding of the planar waveguides. The overlap of the zero-order mode to the fluid is roughly $20 \%$, i.e., the fraction of the energy that resides in the fluid compared to the total mode energy. The total interaction with the fluid (losses due to the evanescent tail probing the fluid) can be increased by fabricating a longer path length. The optical waveguides are customarily used in a spiral form to minimize the area used on the chip.

The sensors described in this work were designed in cooperation with LioniX to record differential on-chip NIR (0.94 to $1.04 \mu \mathrm{m})$ absorption. Waveguides with equal sensing areas are used to probe the absorption difference between two fluids contained in the microfluidic channels. The reference 
channel contains water and the sample channel contains water with ionic content. In Fig. 2 a diagram of the optofluidic sensors is presented.

This work presents cross-section optimizations of a silicon nitride $\left(\mathrm{Si}_{3} \mathrm{~N}_{4}\right)$ core embedded in silicon oxide $\left(\mathrm{SiO}_{2}\right)$ to enable single mode transverse electrical (TE) light propagation (0.94 to $1.04 \mu \mathrm{m}$ ) having high sensitivity, high tolerance for fabrication deviations and $<0.01 \mathrm{~dB} / \mathrm{cm}$ bend losses for a radius of $500 \mu \mathrm{m}$. Furthermore, path lengths for $0.1,0.5$ and $1.5 \mathrm{~dB} / \mathrm{cm}$ propagation losses of waveguides were calculated to obtain the maximal differential signals; and were used in designs varying in sensitivity and complexity. Design of an optofluidic sensor based on a differential absorbance method to detect ionic content in water is not published before, as far as the authors are aware of.

\section{CROSS-SECTION OPTIMIZATION}

The simplest geometry of the TriPleX technology was chosen to propagate the NIR light in the chips: a $\mathrm{Si}_{3} \mathrm{~N}_{4}$ core surrounded by a $\mathrm{SiO}_{2}$ cladding, henceforth called buried structure. The top cladding of the buried structure was removed by chemical etching to probe the fluid with the evanescent field in the sensing area of the chip, henceforth called sensing structure. The cross-sections of the buried and sensing structure are shown in Fig. 3. The figure also shows a simplified cross-section of an over-etched sensing structure.

The thickness of the $\mathrm{Si}_{3} \mathrm{~N}_{4}$ core can be well controlled $( \pm 2$ $\mathrm{nm}$ ) with the etching steps in the fabrication process, whereas the width of the waveguides depends on the lithography deviating approximately $10 \%$. Over-etch depths are estimated to be in the range of 50 to $150 \mathrm{~nm}$ based on a previous fabrication run. The thickness $(t)$ and width $(w)$ were optimized to obtain the following features for the broadband wavelength $(\lambda)$ range:

- A single mode operation,

- high sensitivity combined with,

- reasonable tolerance for fabrication deviations,

- and $<0.01 \mathrm{~dB} / \mathrm{cm}$ bend losses for a radius of $500 \mu \mathrm{m}$.

The Film Mode Matching (FMM) method was used to determine the mode profiles propagation through the different structures (sensing and buried). Sensitivity $(S)$ of differently propagating modes can be expressed by the ratio of the mode energy in the top cladding and the energy of the entire mode propagating through the structure. The sensitivity was calculated by determining the change in the effective refractive index due to a small induced change in refractive index of the fluid for the sensing structure. Likewise, the sensitivity of light propagating through the buried structure due to strain or heat on the top cladding was calculated by determination of the change in the effective refractive index due to the change in the refractive index $(n)$;

$S(\lambda, t, w)=\left[n_{e f f}(n)-n_{e f f}\left(n+\Delta_{n}\right)\right] \Delta_{n}{ }^{-1}$

where the effective refractive index $\left(n_{\text {eff }}\right)$ was determined by the FMM method in "Optodesigner" (Phoenix) for the sensing structure with and without a small addition $\left(\Delta_{n}\right)$ to the refractive index of the fluid or top cladding in case of the buried structure.

The bend losses were determined by the Finite Difference (FD) method. The calculation of losses with the FD method requires more computational power compared to the FMM method. The mode is barely guided for small sizes of the $\mathrm{Si}_{3} \mathrm{~N}_{4}$ core, leading to low sensitivity and high bend losses. Therefore, the FMM method was first utilized to determine the parameters, i.e., the range of sizes of the $\mathrm{Si}_{3} \mathrm{~N}_{4}$ that lead to single mode propagation with at least $17 \%$ sensitivity. The effective index method (EIM) was used to determine the effective refractive index for wavelengths with $0.01 \mu \mathrm{m}$ increments ranging from 0.9 to $1.08 \mu \mathrm{m}$ of the TE0 mode for both the sensing and buried structures.

The methods are implemented using the 64-bit version of Optodesigner, licensed by Phoenix. The data were saved (with 15 significant digits) to a solid state drive. The software evaluated 300 different modes in the FMM method to ensure that the guided modes were found. Convergence was verified for the parameters used (grid-size, number of modes, etc.).

The refractive indices at room temperature $\left(20^{\circ} \mathrm{C}\right)$ for water, thermally grown silicon dioxide, single-crystal silicon and low-pressure chemical vapor deposition (LPCVD) stoichiometric silicon nitride were provided by LioniX. The sensitivity of the propagating mode of the buried structure was determined for the different sizes of the $\mathrm{Si}_{3} \mathrm{~N}_{4}$ layer and is presented by color coding in Fig. 4. The waveguide dimensions can be defined for single mode operation from this figure.

The sensitivity was determined for three wavelengths 0.94 , 0.99 and $1.04 \mu \mathrm{m}$ to define the spectral response within the $100 \mathrm{~nm}$ bandwidth of the sensor. The sharp cut-off in sensitivity seen in the $2 \mathrm{D}$ color-coded figure of the $\mathrm{TE}_{1}$ mode gives a visual impression of the size of the $\mathrm{Si}_{3} \mathrm{~N}_{4}$ for which the mode becomes guided.

The sensitivity of the $\mathrm{TE}_{0}$ and $\mathrm{TE}_{1}$ modes calculated for the sensing structure is presented in Fig. 5 for wavelengths 0.94 $\mu \mathrm{m}, 0.99 \mu \mathrm{m}$ and $1.04 \mu \mathrm{m}$, respectively, in three distinct situations: perfect removal of the top cladding, $100 \mathrm{~nm}$ overetch and $200 \mathrm{~nm}$ over-etch of the top cladding. Light of shorter wavelengths is better confined than the light of longer wavelengths which can be seen in the figure. The increase in sensitivity due to a deeper over-etch is visible in the first order mode for a wavelength of $0.94 \mu \mathrm{m}$.

Two suitable combinations of height and width that display high sensitivity and high foundry tolerance in the width of the $\mathrm{Si}_{3} \mathrm{~N}_{4}$ core were found for single mode propagation based on the data presented in Figs. 4 and 5. The first combination has a width of $1.95 \mu \mathrm{m} \pm 0.15 \mu \mathrm{m}$ and a thickness of $83 \mathrm{~nm} \pm 2 \mathrm{~nm}$ and the second combination has a width of $1.7 \mu \mathrm{m} \pm 0.1 \mu \mathrm{m}$ and a thickness of $92 \mathrm{~nm} \pm 2 \mathrm{~nm}$, respectively. These combinations balance fabrication tolerance in the width against bend losses.

\section{A. Bend losses}

The losses due to bend radii of 150, 333 and $500 \mu \mathrm{m}$ were determined for the $\mathrm{TE}_{0}$ mode with wavelengths of 0.94, 0.99 and $1.04 \mu \mathrm{m}$ for the sensing structure with 0,50 and $150 \mathrm{~nm}$ 
over-etch and the sizes of the two regions. The spectral absorption of water was included in the simulation to determine the band losses for a sensing structure with a $\mathrm{Si}_{3} \mathrm{~N}_{4}$ core thickness of $92 \mathrm{~nm} \pm 2 \mathrm{~nm}$ and width of $1.6 \mu \mathrm{m} \pm 0.2 \mu \mathrm{m}$. The spectral losses due to water of a straight sensing waveguide were subtracted to determine the losses due to bend radii.

Fig. 6 shows the bend losses for a $\mathrm{TE}_{0}$ mode propagating through a sensing structure with a $\mathrm{Si}_{3} \mathrm{~N}_{4}$ core of $1.6 \mu \mathrm{m}$ by 92 $\mathrm{nm}$. The $<0.01 \mathrm{~dB} / \mathrm{cm}$ bend losses for a radius of $500 \mu \mathrm{m}$ can be achieved for the entire spectral range and an over-etch depth of $50 \mathrm{~nm}$. The sizes of the $\mathrm{Si}_{3} \mathrm{~N}_{4}$ core in the second region led to ten times higher bend losses. Therefore, the parameters of the first region were used for the chip fabrication although the parameters of the second region would give an additional $0.1 \mu \mathrm{m}$ foundry tolerance in the width of the $\mathrm{Si}_{3} \mathrm{~N}_{4}$ core.

The fraction of the mode overlaps with the fluid increases with over-etch; and bending of a waveguide leads to spatial shift of the propagating mode (e.g., a bend to the left would cause a shift of the mode to the right, deeper into the fluid). The increase of mode sensitivity to the water at a certain radius is therefore expected to increase with the over-etch depth; and with that, the losses are expected to increase. Fig. 6 confirms that the deeper over-etch leads to a higher bend loss.

The bend losses of the shorter wavelengths are smaller than those of the longer wavelengths in the absence of absorption due to better confinement of the light. Additional loss originates from the second overtone of the $\mathrm{OH}$ vibration, whose absorption maximum lies at $0.97 \mu \mathrm{m}$ and falls off steeply to either side.

\section{SENSING LENGTH}

The transmission difference due to the presence of ions in water is small; only approximately $0.002 \cdot c$ of the total intensity for a $1 \mathrm{~cm}$ optical path, where $c$ is the concentration of ions in mol/L. The intensities of the differential transmission $\left(I_{S}\right)$ for different path lengths can be calculated using the Beer-Lamberts law [12]:

$I_{s}=10^{-A_{\text {water }}}-10^{-A_{\text {water }}[1 \pm(\Delta \cdot c)]}$

where $A_{\text {water }}$ is the spectral absorption of demineralized water, $\mathrm{c}$ is the concentration in $\mathrm{mol} / \mathrm{L}$ and $\Delta$ is the unique spectral effect (fingerprint) which the ions exert on the absorption spectrum of water. For $0.97 \mu \mathrm{m}$ this $\Delta$ is in the order of 0.04 for most electrolytes, and the optical density of water for this wavelength is approximate 0.2. This simplifies (2) to define the differential transmission by the influence of both the geometrical path length $(L)$ and the concentration of ions:

$I_{s}=10^{-0.2 L}-10^{-0.2 L[1 \pm(0.04 \cdot c)]}$.

The detection limit of a sensor based on differential absorbance is proportional to its dynamic range and can be maximized by optimizing the optical path length.

Fig. 7 presents normalized calculated differential signal intensities plotted against the sensing length for different concentrations, $0.5,0.25,0.13,0.06$ and $0.03 \mathrm{~mol} / \mathrm{L}(\mathrm{M})$, respectively. The concentration of the electrolytes exerts an effect on the normalized signal as one would expect; however, the optimum sensing length for these concentrations seems to be not significant affected. The optimum length $\left(L_{m}\right)$ can be obtained by solving the derivative of $I_{s}$ with respect to $L$ and setting the derivative to zero:

$\frac{d I_{S}}{d L}=\frac{(c+25)}{125} \cdot \log _{e}(10) \cdot 0.1^{\frac{(c+25)}{125}}-0.2 \log _{e}(10) \cdot 0.1^{0.2 L}$
$L_{m}=(125 / c) \cdot \log _{10}[1+(c / 25)]$.

The optimum geometrical length (5) for differential absorption measurements between two similar fluids is approximately $2.17 \mathrm{~cm}$ for lossless propagation through air, mirrors and cuvettes. This path length leads to a six fold increase of the differential transmission compared to a path length of $1 \mathrm{~cm}$. To translate the optimum geometric path length through a cuvette to the optimum integrated geometric sensing path length on the optical chips without waveguide losses (due to the roughness of $\mathrm{Si}_{3} \mathrm{~N}_{4}$ layer, absorption losses due to $\mathrm{SiO}_{2}$ and $\mathrm{Si}_{3} \mathrm{~N}_{4}$, etc.). The result of (5) is multiplied by five to account for the $20 \%$ fraction of the propagating mode in the fluid in the sensing windows.

The optimum sensing length is independent of the electrolyte concentration due to the small effect on the absolute absorption as can be seen in Fig. 8. A model that includes the waveguide losses is shown in Fig. 9. Incorporation of these losses significantly reduces the optimum sensing length. The silicon-based platform TriPleX allows low-loss propagation and small bend radii. For example, a loss of $0.095 \mathrm{~dB} / \mathrm{cm}$ was recorded at a waveguide radius of $70 \mu \mathrm{m}$ for light with a wavelength of $1.55 \mu \mathrm{m}$ [13]. However, the etching required for sensing leads to the higher surface roughness of the waveguides and therefore also higher propagation losses are expected. Three sensing lengths were chosen to account for different propagation losses 2.5, 5 and $7.5 \mathrm{~cm}$.

\section{INTERFEROMETRIC DETECTION}

Interferometric mixing was explored in different designs to improve the detection limit of the signal. A Mach Zehnder (MZ) interferometer with a $\pi$ phase difference between its two arms would produce complete destructive interference on one output (the 'dark' port). Half of the intensity difference in transmission of both arms, due to a difference in absorption, is directly measurable at the dark port. Naturally, ions in water influence both the real and imaginary parts of the complex refractive index of water.

Therefore, not only a perfect 50/50 splitting ratio of the coupling devices is required with a broadband $\pi$ shifter, but also the difference in the real part of water with that of an electrolyte needs to be compensated. Heaters are positioned above the buried waveguide and used to affect the effective refractive index of the waveguide (in the reference path) to compensate the difference in dispersion due to the presence of ions in the sample path. The increase of optical path length due to the presences of ions can be compensated by increasing the temperature of the $\mathrm{SiO}_{2}$ in the other interferometric arm, 
thereby increasing its optical path length. The compensation can also be achieved by a temperature offset between the reference and sample fluids. But, the change in spectral absorbance of the water due to this temperature difference needs to be accounted for [14],[15].

Differences in path length can be realized in other configurations of the MZ and so different number of spectral fringes can be recorded at the output ports of the MZ. The spectral fringes are influenced by the spectral phase relation between the two arms of the MZ. Fringe resolution is dependent in part on the modulation depth (i.e., the contrast on the chip). The number of fringes is linearly dependent on the change of the refractive index and the optical path length difference. A change in fringe spacing can be used to quantify the ionic concentration. The shape of the spectral envelop allows the identification of the ionic constituents by differential absorption [16],[17].

The deepest point of the destructive interference depends on the number of fringes in the spectral region in combination with the spectral resolution of the recording instruments. It is essential to accurately measure the depth, as it signifies the absorption imbalance. To accurately record the modulation two approaches were taken. The coherence length of the laser and the spectral resolution of a spectrometer limit the fringe depth. Ionic content will reduce fringe depth. Hence, we offset the optical path by three separate designs to compensate for low $(60 \mathrm{mM})$, medium $(0.5 \mathrm{M})$ and high $(1 \mathrm{M})$ ionic concentrations. Differences in path length were chosen to enable 10, 27 and 53 fringes in the spectral region. And secondly, heaters in the buried structure were used to shift the optical path length at least one full fringe. The first approach requires a spectrometer with a high spectral resolution but simplifies chip design.

The coherence length of the laser should be considered when determining the differential absorbance in both approaches. The following equations were used to determine suitable path difference of the buried waveguides between the two arms:

$\Delta_{M Z}(\lambda)=n_{r b} L_{r b}-n_{s b} L_{s b}+n_{r f} L_{r f}-n_{s f} L_{s f}$

and

$\Delta_{M Z}^{g}(\lambda)=N_{r b} L_{r b}-N_{s b} L_{s b}+N_{r f} L_{r f}-N_{s f} L_{s f}$,

where $L$ is the geometric path length, $n$ the effective refractive index and $N$ the group effective refractive index. The subindices $r b, s b, r f$ and $s f$ signify reference buried, sample buried, reference fluid and sample fluid, respectively. The path lengths of both sensing waveguides were set to be of the same length. A phase shift was induced by designing a difference in the length of the buried waveguides. The spectral signal $\left(\mathrm{S}_{\mathrm{MZ}}\right)$ can be calculated using

$S_{M Z}(\lambda)=y\left(1+V \cos \left\{2 \pi \Delta_{M Z}(\lambda) / \lambda\right\} \exp \left\{-0.5\left[\pi \Delta_{M Z}^{g} \Delta_{R} /\right.\right.\right.$

$\left.\left.\lambda]^{2}\right\}\right)$

where the width of the spectrometer response function $\Delta_{R}$ is set to $0.23 \cdot 10^{-3} \mu \mathrm{m}, V$ is the visibility term and $y$ is the normalized spectral source. A path length difference of 0.3 , 59.5, 150 and $300 \mu \mathrm{m}$ leads to $0,10,27$ and 53 spectral fringes, respectively.

\section{V. $\Xi$ COUPLER}

A $\Xi$ coupler is a bidirectional coupler with an extra waveguide in the middle. It does not exhibit the disadvantages of using $y$-splitters for recombination of light, such as the inherent loss and a single output port. The dark output port of a y-splitter is the unguided propagation mode in the cladding, inaccessible to record. A directional coupler mimics the free space beam splitter with its two output ports. However, the intensity and phase relationship between the output ports is dissimilar to the free space component; the intensities at the output ports of the coupling device are dependent on spectral input and geometric parameters the coupling waveguides.

The symmetric design of the $\Xi$ coupler yields three in- and outputs ports with equal power at the outer output ports when the light is coupled to the middle input port. However, the light intensity at the outer waveguides depends on the coupling length and spectral input. The phase relation can be kept constant by adiabatic coupling as shown in Fig. 10. Thus leading to a flattened intensity response to both the length of coupling and wavelength. Both y-splitters and $\Xi$ coupler were incorporated in the chip design to achieve achromatic bisection of light.

To evaluate the light propagation through the $\Xi$ coupler the beam propagation method (BPM) was used to obtain the percentage of light propagating in the different waveguides due to the relevant parameters $(d W, L$ and $\lambda$ ) of the $\Xi$ coupler. Fig. 11 shows the light propagation dependence through a $\Xi$ coupler with different coupling length, gap size, bending length and change in width. The $\Xi$ coupler was designed for the wavelength region of interest, based on designs presented by Hsien-kai Hsiao, et al. [18]. Table 1 shows the parameters used for the different $\Xi$ couplers in the chip design.

\section{DESIGNS}

Fig. 12 shows an overview of the schematic designs. Design 11,12 , and $13\left(\mathrm{D}_{11-13}\right)$ contain electronic subtraction schemes, whereas the other designs are interferometric to perform the subtraction optically. Table I presents the parameters of the $\Xi$ coupler used in design $\mathrm{D}_{21-23}$ and $\mathrm{D}_{41-43}$. $\mathrm{D}_{21-23}$ replaces the $\mathrm{y}$ splitter with a $\Xi$ coupler to enable a Michelson interferometer on chip reducing the required sensing path by a factor of 2 . The difference in path length was 59.5 and $150 \mu \mathrm{m}$ for the two Michelson interferometers placed on each chip. Designs $\mathrm{D}_{31 \mathrm{a}-}$ 34a feature a Mach Zehnder layout with a path difference of $0.3,59.5,150$ and $300 \mu \mathrm{m}$, respectively. Design $\mathrm{D}_{31 \mathrm{~b}}$ - D34b utilizes a homodyne interferometric layout and design $\mathrm{D}_{41}$ - D43 used an identical layout of the Mach Zehnder, but has a $\Xi$ coupler to recombine both light paths. $D_{51}$ and $D_{52}$ were designed to enable online calibration of the sensor.

\section{FABRICATION}

The chips were fabricated by Lionix according to the designs specifications of this work and allowed single mode propagation of the designed spectral bandwidth. To ease the 
effort of aligning the fiber array to the chip gold lines were deposited on top of the chip, located between the waveguides, to visually mark the position of the embedded waveguides. The etching area was chosen to be as small as possible to avoid an inconsistent top layer close to the interface of buried and sensing structure, i.e., the top cladding above the spirals was removed instead of the entire window.

\section{CONCLUSIONS}

Multiple approaches to measuring differential transmission caused by the presence of ions in water have been presented. They may also be applied to different applications where small differences in absorption need to be measured between fluids of similar composition. It is feasible to design and simulate a differential absorption based optofluidic sensor for determination of ionic content in water. The parameters leading to a $100 \mathrm{~nm}$ single mode wavelength region with bend losses lower than $0.02 \mathrm{~dB} / \mathrm{cm}$ and a sensitivity around $20 \%$ whereas keeping the reasonable foundry tolerances were presented.

Moreover, the optimal sensing arm length was found to be independent of the concentration of ionic content due to small differences in transmitted intensity but does depend on the losses of the waveguides. Adjustments of the sensing length can be designed to compensate for these losses.

Interferometric techniques permit determination of the differential complex refractive index between two fluids and can be used to measure differential absorption signal on-chip.

Finally, the adiabatic $\Xi$ coupler with its flattened spectral response could be an interesting tool for interferometric designs as has been shown for a Michelson interferometer.

\section{OUTLOOK}

The initial characterization results of the fabricated chips are promising (Fig. 13). A representative example for the realization of one of these design is given in Fig. 14. It shows the spectral output of a Mach Zehnder $\left(\mathrm{D}_{32 \mathrm{a}}\right)$ recorded by an Avantes spectrometer (AvaSpec-ULS3648 StarLine Highresolution). The shifting of fringes was recorded for an applied potential of $20 \mathrm{~V}$ and a current ranging from $0 \mathrm{~mA}$ to $40 \mathrm{~mA}$. Stray light detected by the spectrometer was responsible for the observed second spectral modulation. Further work will involve the recording of the spectral output of the different designs with different concentrations of ionic species in the fluidic channel.

\section{ACKNOWLEDGMENT}

This work was performed at Wetsus, European Centre of Excellence for Sustainable Water Technology (www.wetsus.nl). Wetsus is funded by the Dutch Ministry of Economic affairs, the European Union Regional Development Fund, the Province of Fryslân, the City of Leeuwarden and the EZ/Kompas program of the "Samenwerkingsverband NoordNederland". The authors like to thank the participants of the research theme "Sensoring" for the fruitful discussions and their financial support.

\section{REFERENCES}

[1] J. Plant. Pollutans, human health, and the environment: a risk based approach. Wiley-Blackwell, Chichester, West Sussex, U.K. Hoboken, N.J, 2012.

[2] P.H. Gleick. Water and terrorism. Water policy, 8:481-503, 2006.

[3] WHO Drinking Water Quality Committee. Water Sampling and Analysis. Guidelines for drinking water quality, 2011, 4th ed.:51-72.

[4] J. P. Murrihy, M. C. Breadmore, A. Tan, M. McEnery, J. Alderman, C. O’Mathuna, A. P. O’Neill, P. O’Brien, N. Advoldvic, P. R. Haddad, and J. D. Glennon. Ion chromatography on-chip. Journal of Chromatography A, 2001, 924(1):233 - 238. 14th International Symposium on Microscale Separations and Analysis.

[5] J. Raich. Review of sensors to monitor water quality. European Commission Joint Research Centre, JRC85442, 2013.

[6] R. Suhrmann and F. Breyer, Untersuchungen im ultraroten absorptionsspektrum über die Änderung des lösungsmittels durch die gelöste substanz. i. uber den einfluss gelöster salze auf den assoziationszustand des löstenden wassers, Z. Physik, Chem. B, 1933, 20(17):17-53.

[7] G. R. Choppin and K. Buijs. Near-infrared studies of the structure of water. ii. ionic solutions. The Journal of Chemical Physics, 1963, 39(8):2042-2050.

[8] J. Lin, J. Zhou and C. W. Brown, Identification of Electrolytes in Aqueous Solutions from Near-IR Spectra. Applied Spectroscopy, 1996, 50:444-448.

[9] J. Lin and C. W. Brown, Spectroscopic measurement of $\mathrm{NaCl}$ and seawater salinity in the near-IR region of 680-1230 nm. Applied Spectroscopy, 1993, 47:239-241.

[10] G. W. Steen, E. C. Fuchs, A. D. Wexler, and H. L. Offerhaus. Identification and quantification of 16 inorganic ions in water by Gaussian curve fitting of near-infra red difference absorbance spectra. Applied Optics, 2015, 54(19):5937-5942.

[11] G. Steen, A. D. Wexler, and H. L. Offerhaus. Optofluidic interferometry chip designs of differential NIR absorbance based sensors for identification and quantification of electrolytes. In Advanced Photonics 2016 (IPR, NOMA, Sensors, Networks, SPPCom, SOF), 2016, page SeW3D.2. Optical Society of America.

[12] A. Beer, Bestimmung der absorption des rothen lichts in farbigen flüssigkeiten, Annalen der Physik, 162(5):78-88, 1852.

[13] L. Zhuang, D. Marpaung, M. Burla, W. Beeker, A. Leinse and C. Roeloffzen, Low-loss, high-index-contrast Si3N4/SiO2 optical waveguides for optical delay lines in microwave photonics signal processing, Optics Express, 19(23):23162-23170, Nov 2011.

[14] K. Wallace, G. Hardy, and E. Serabyn, Deep and stable interferometric nulling of broadband light with implications for observing planets around nearby stars. Nature, 406(6797):700-702, 2000.

[15] M. R. Bolcar and R. G. Lyon, Approaches for achieving broadband achromatic phase shifts for visible nulling coronagraphy, 2012.

[16] P. Hlubina, M. Kadulová, and D. Ciprian. Spectral interferometry-based chromatic dispersion measurement of fibre including the zero-dispersion wavelength. Journal of the European Optical Society - Rapid publications, 7(0), 2012.

[17] B. Anderson, E. Bernhardt, and M. G. Kuzyk, A white light interferometric microscope for measuring dose-dependent reversible photodegradation, Journal of Applied Physics, 114(12):123103, 2013.

[18] H. K. Hsiao, K. A. Winick, J. D. Monnier, and J. P. Berger, Integrated optic beam combiners for stellar interferometry and nulling at near- and mid-infrared wavelengths, 2010. 


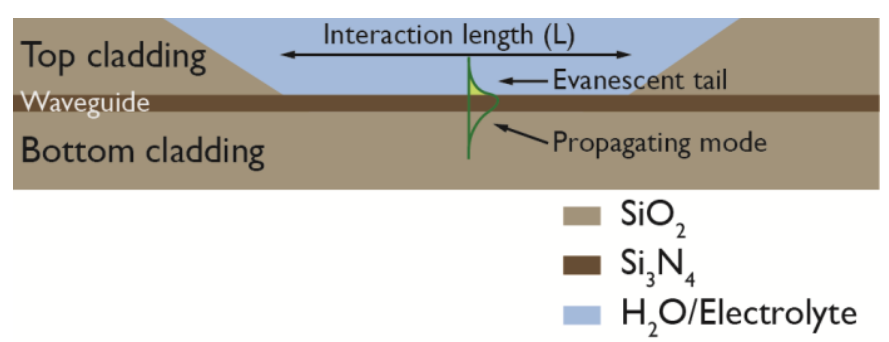

Fig. 1. Schematic of the longitudinal section of the sensing window. Light is confined by the thin layer of $\mathrm{Si}_{3} \mathrm{~N}_{4}$ laying between a cladding of $\mathrm{SiO}_{2}$ on the wafer (silicon substrate). The top cladding of the TriPleX configuration is removed to enable sensing by interaction of the evanescent tail with the tail.

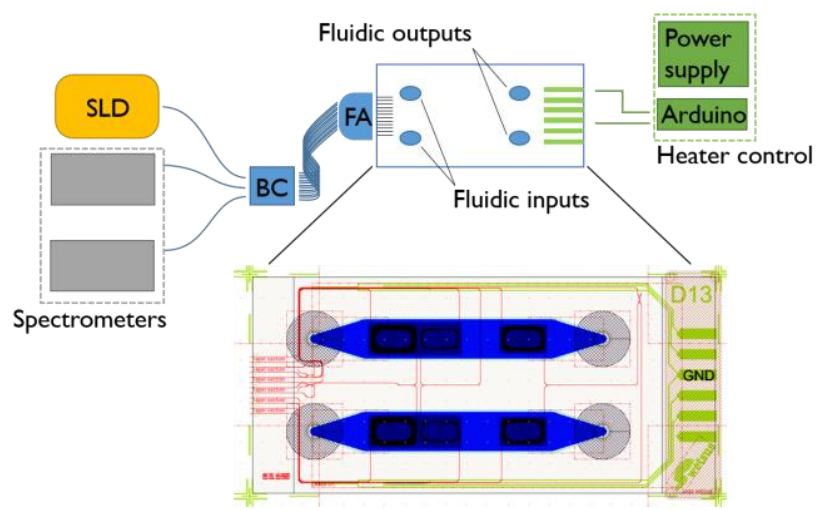

Fig. 2. Schematic of the setup. Light from a broadband light source (SLD) is butt-coupled (BC) to a fiber array (FA) and guided to the chip. The blue color in the zoom-in shows the microfluidic channel. The top cladding is removed to enable sensing in the dark blue region. The output light is coupled back to the FA and read by the spectrometers. The green lines depict the electrical connections to the heaters.
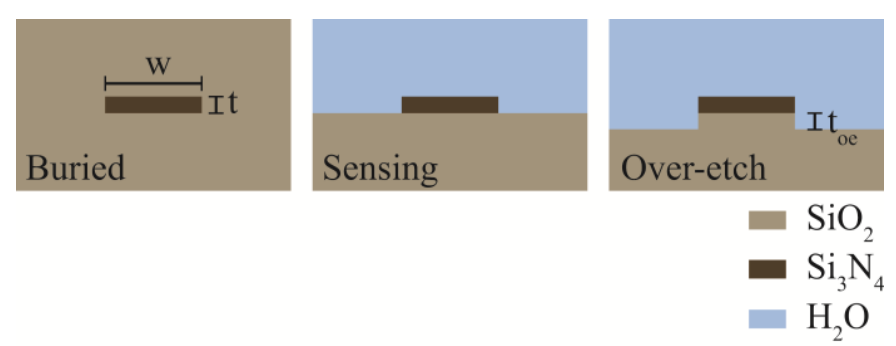

Fig. 3. Schematic representations of different chip structures visualized with the geometric parameters: width $(w)$, thickness $(t)$, and over-etch depth $\left(t_{o e}\right)$.

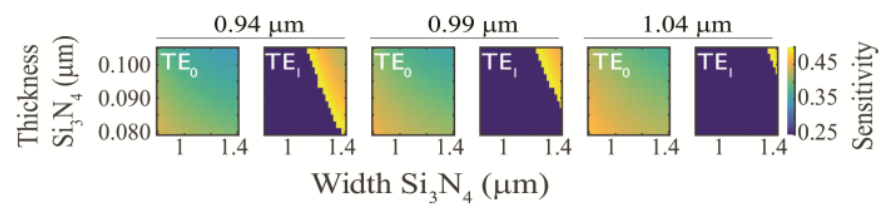

Fig. 4. Color coded sensitivity of the $\mathrm{TE}_{0}$ and $\mathrm{TE}_{1}$ modes calculated for the buried geometry with the center wavelength and the outer wavelengths.

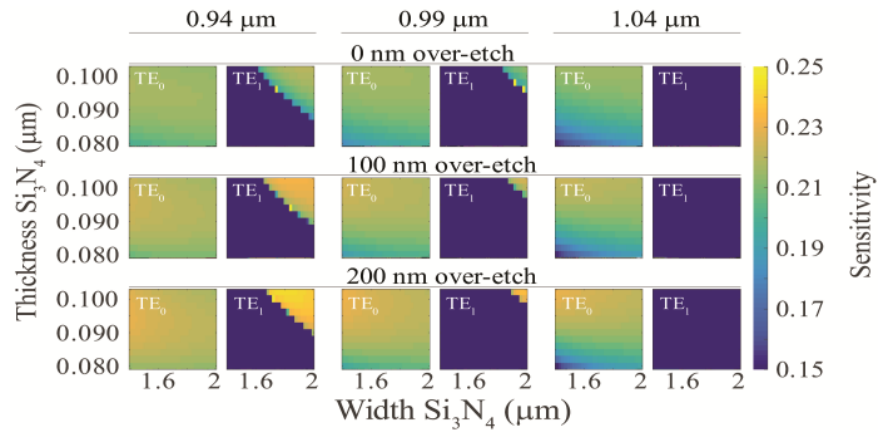

Fig. 5. Color coded sensitivity of the $\mathrm{TE}_{0}$ and $\mathrm{TE}_{1}$ modes calculated for the sensing geometry with $0 \mathrm{~nm}, 100 \mathrm{~nm}$ and $200 \mathrm{~nm}$ over-etch depth.

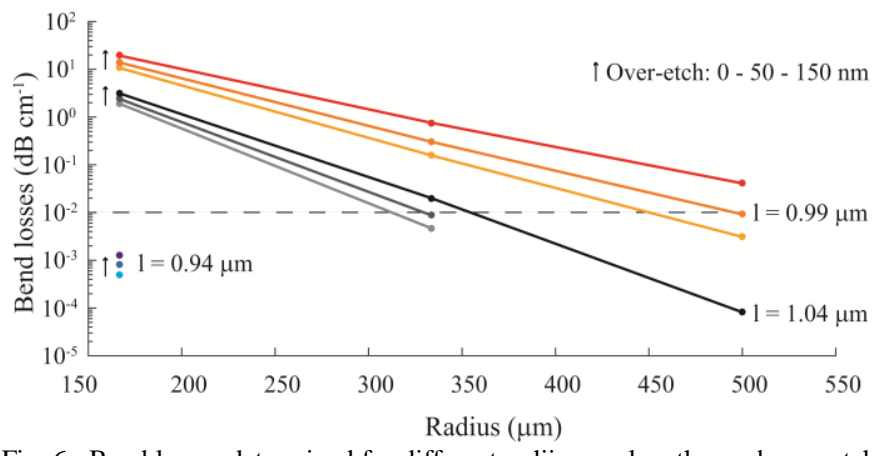

Fig. 6. Bend losses determined for different radii, wavelengths, and over-etch depths. The dashed line indicates the desired $<0.01 \mathrm{~dB} / \mathrm{cm}$ bend losses for a radius of $500 \mu \mathrm{m}$. Due to the FD limitation of the simulations only the bend losses for a radius of $166 \mu \mathrm{m}$ are displayed for wavelength $0.94 \mu \mathrm{m}$.

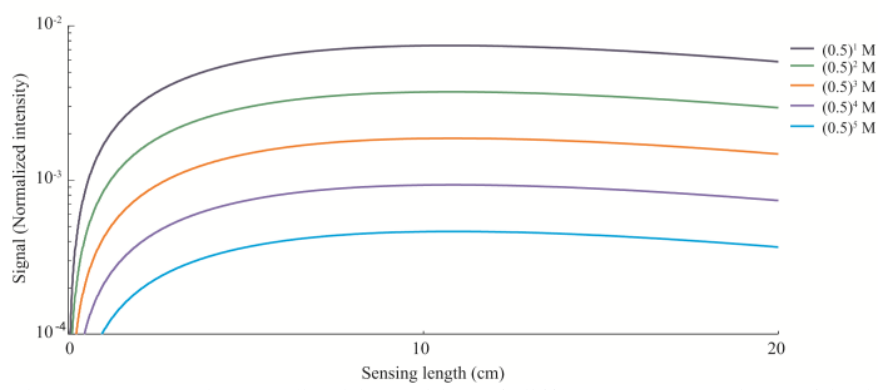

Fig. 7. Expected normalized intensities of different concentrations of ionic content against sensing lengths when $20 \%$ of the propagating light is exposed to the fluid.
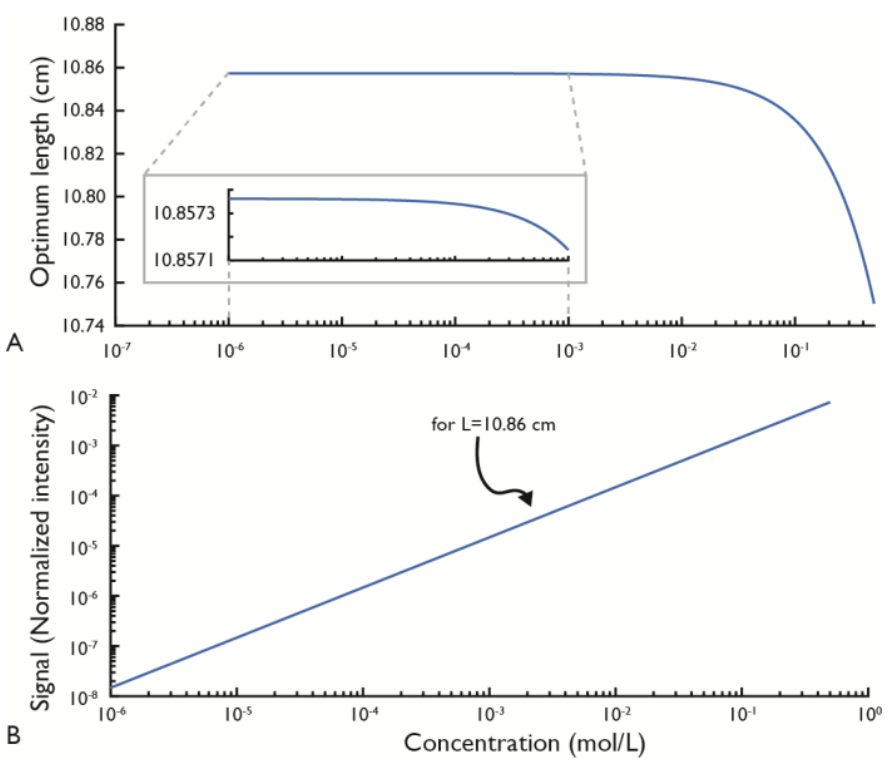
Fig. 8. (A) Optimum integrated geometric sensing path length against electrolyte concentration. (B) Expected normalized signal against concentration for an optimized length of $10.86 \mathrm{~cm}$.

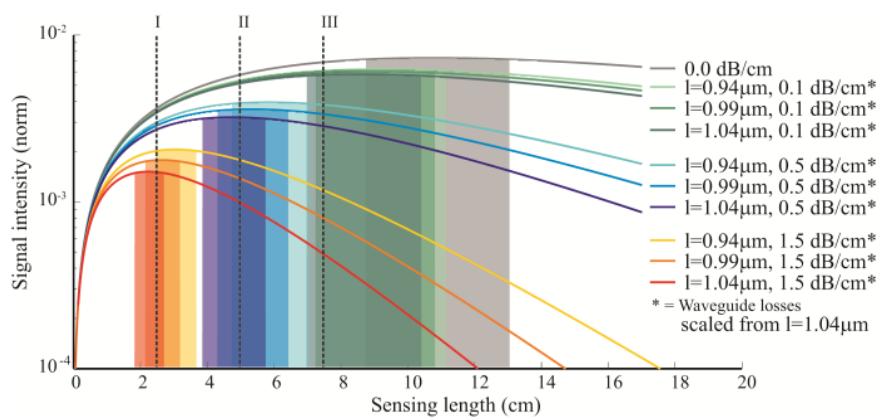

Fig. 9. The expected normalized intensity of a $0.5 \mathrm{M}$ electrolyte is plotted against sensing length. The gray line presents a waveguide without losses; the green, blue and orange lines present waveguides with losses of $0.1 \mathrm{~dB} / \mathrm{cm}, 0.5$ $\mathrm{dB} / \mathrm{cm}$ and $1.5 \mathrm{~dB} / \mathrm{cm}$ at $\lambda=1 \mu \mathrm{m}$, respectively. The wavelength dependent losses are calculated with the scaling factor $\left(\lambda_{0} / \lambda_{1}\right)^{4}$. Sensing lengths chosen for chips designs are shown as dotted lines I, II and III plotted at 2.5, 5.0 and $7.5 \mathrm{~cm}$, respectively. The shaded areas represent $95 \%$ of the signal intensity for a given set of parameters. Short sensing lengths are appropriate for high waveguide losses.

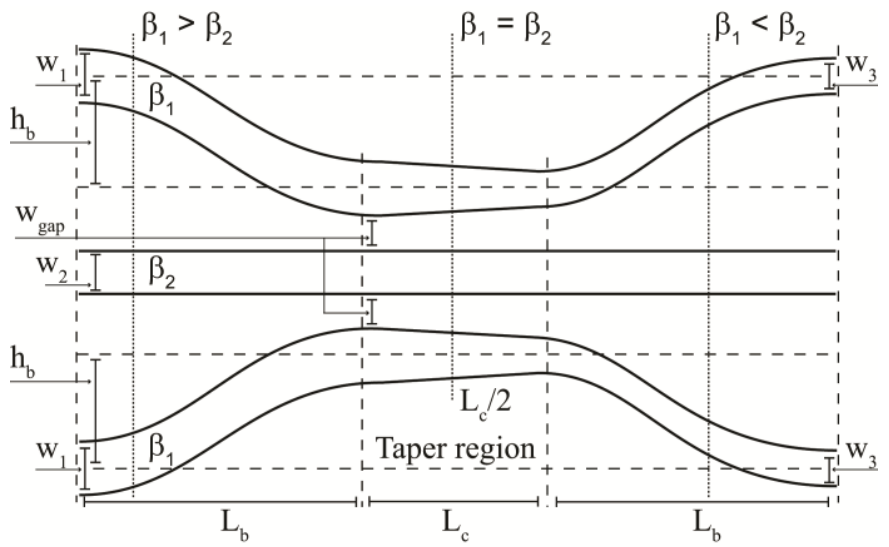

Fig. 10. The $\Xi$ coupler with relevant parameters: the width of the gap $\left(w_{g a p}\right)$ of $1 \mu \mathrm{m}$, the width of the outer waveguides $\left(w_{l}\right)$ equal to the width of the middle waveguide $\left(w_{2}\right)$ plus a delta width $\left(\Delta_{W}\right)$ before tapered to $w_{2}$ minus the $\Delta_{W}$, the height of the bend $\left(h_{b}\right)$ equal to $5 \mu \mathrm{m}$, the length of the bend $\left(L_{b}\right)$ and the length of the coupling $\left(L_{c}\right)$.

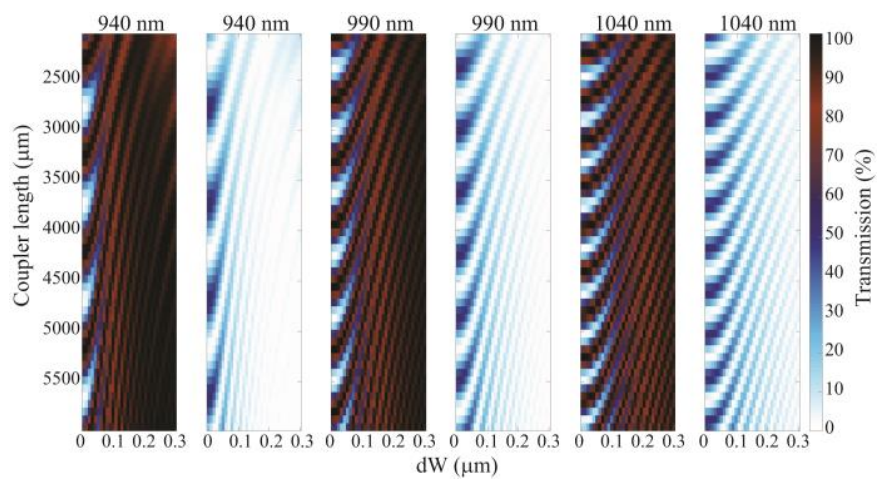

Fig. 11. The left graph (darker) and right graph (brighter) present the percentage of light coupling to the outer waveguides and middle waveguide of the $\Xi$ coupler, respectively for three different wavelengths. The percentage was color coded from numerical values obtained from the BPM simulations. The coupler length of $\Xi$ coupler is varied on the y-axis and the differential width of the outer waveguides is varied on the $\mathrm{x}$-axis.
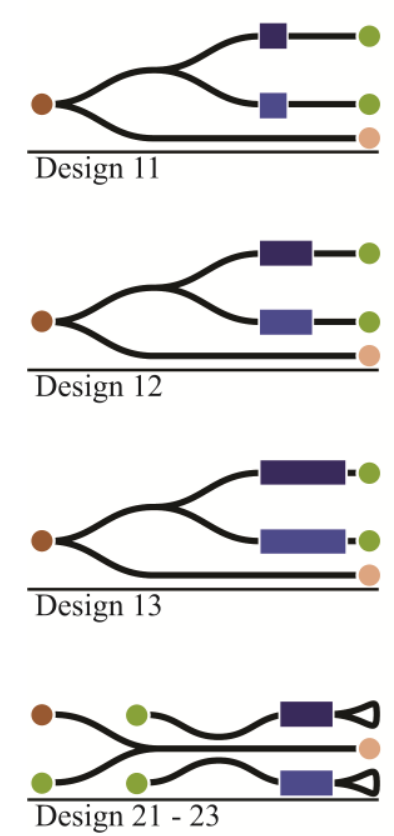

— Waveguide

- SM Fiber (out)

- PM Fiber (in)

SM Fiber (ref)

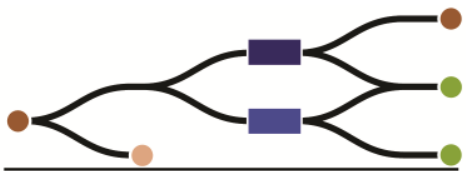

Design 31a - 34a

Fig. 12. Five designs are schematically presented. The heaters are not drawn for simplicity. Design 11-13 ( $\left.\mathrm{D}_{11-13}\right)$ are placed upon one die (chip). Similar, two Michelson interferometers $\left(\mathrm{D}_{21-23}\right)$ are placed on one die. The chips were designed for one standardized fiber array (3 PM fibers and 9 SM fibers) to minimize the cost. Every design exhibits fluidic windows for sensing of the sample and the reference fluid water (dark- and light blue, respectively). The design complexity varies to enable different purposes. Design 1 is based on the non-interferometric method of detection, Design 2 is a Michelson interferometer (reducing the required path length by two). Design 3 is a Mach Zehnder interferometer with normal y-splitter. Design 4 combines a Mach Zehnder with a $\Xi$ coupler to reduce optical losses. Design 5 uses a double Mach Zehnder to correct for temperature drifts.
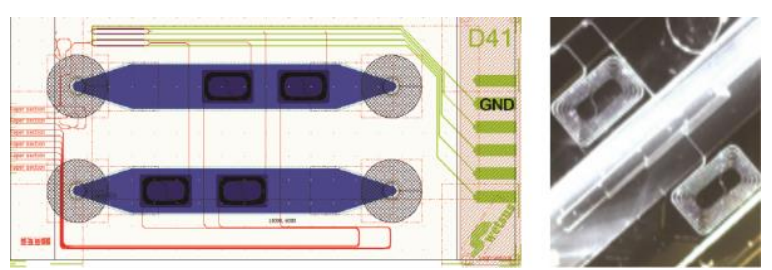

Fig. 13. The realization of a chip. Left) The mask design of optical layout Design 41 and (right) a microscopic image taken of the fabricated chip. 


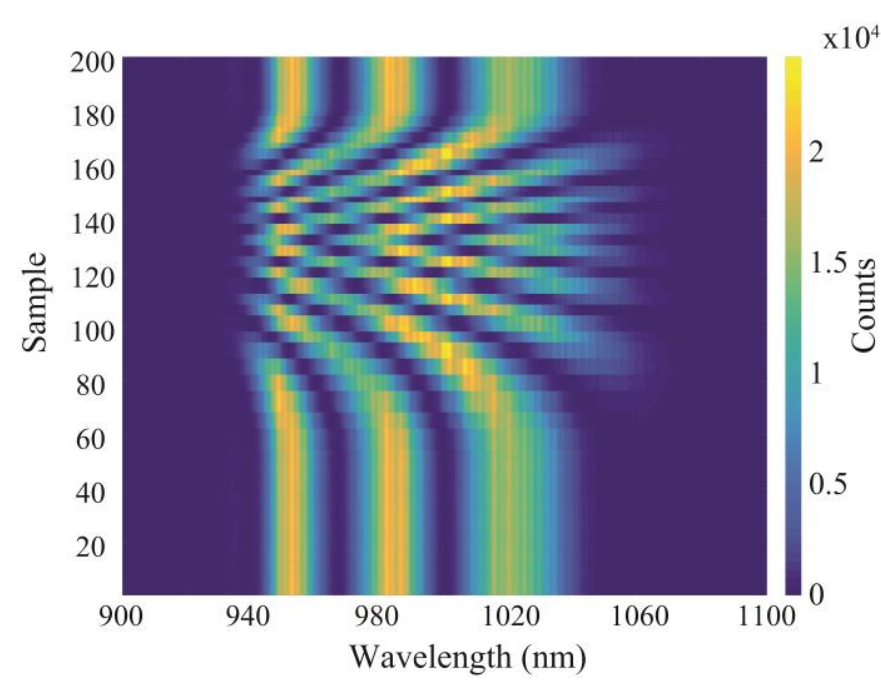

\section{1}

Fig. 14. The current over the heater in one path of the Mach Zehnder is gradually increased to $40 \mathrm{~mA}$ and thereafter decreased to $0 \mathrm{~mA}$ in 100 discrete steps (with an applied potential of $20 \mathrm{~V}$ ). The recorded spectral signal (counts/wavelength) is color coded from the numerical values.

TABLE I

PARAMETERS $\Xi$ COUPLER

\begin{tabular}{ccc}
\hline \hline $\mathrm{dW}(\mu \mathrm{M})$ & $\mathrm{L}_{\text {coupler }}(\mu \mathrm{m})$ & $\mathrm{L}_{\text {bend }}(\mu \mathrm{m})$ \\
\hline
\end{tabular}

